\title{
Enhancing online forms: Use format specifications for fields with format restrictions to help respondents
}

\author{
Javier A. Bargas-Avila*, Sébastien Orsini, Hannah Piosczyk, Dominic Urwyler, Klaus Opwis \\ University of Basel, Department of Psychology, Center for Cognitive, Psychology and Methodology, Switzerland
}

\section{A R T I C L E I N F O}

\section{Article history:}

Received 19 March 2010

Received in revised form 6 August 2010

Accepted 15 August 2010

Available online 20 August 2010

\section{Keywords:}

Online forms

Format restriction

Error prevention

Data entry restriction

User feedback

Form validation

\begin{abstract}
A B S T R A C T
Field format restrictions are often used in online forms to impose certain formatting and content rules on users, such as minimum password length or date entry format. In this study, the question whether and how format restrictions for fields in online forms should be communicated to Internet users was explored. In an online study with $n=166$ participants, four ways to communicate format restrictions were investigated: (1) no visual format restriction, (2) format examples, (3) format specifications, and (4) both format restrictions (examples and specifications). Results show that providing details of any format restriction to users in advance leads to significantly fewer errors and trials. The most efficient way to communicate field format restrictions to users is by stating the imposed rule (format specification). Providing an additional example neither helps nor constrains users.
\end{abstract}

(C) 2010 Elsevier B.V. All rights reserved.

\section{Introduction}

In the past decade, the Internet has changed from a one-way information pool to an interactive social world, where users communicate and transact with other users, institutions and companies. These interactions often involve revealing personal information in online forms, registrations and surveys. Forms are often not adequate for complex human-computer interactions, but work well for simple transactions, when there is not much to do beyond plain data entry (Camenisch et al., 2006; Nielsen, 2005). In this manner, forms enable commerce, communities and productivity on the web to thrive. Forms also stand between users and web applications (Wroblewski, 2008); therefore, web designers may wish to make sure that these forms are perceived by users as efficient, effective and satisfactory.

In online forms, usability plays a major role, because of the somewhat dry nature of filling in forms. Users should be able to complete them as quickly and easily as possible to avoid aborted transactions (Garrett, 2002; Wroblewski, 2008). Good web form design supports error avoidance during data entry. However, errors are often difficult to prevent; when errors occur, it is important to help users to correct them as quickly and easily as possible thus

\footnotetext{
* Corresponding author. Tel.: +4161 2670583; fax: +41612670632.

E-mail address: javier.bargas@unibas.ch (J.A. Bargas-Avila).
}

improving dialogue (Bargas-Avila and Oberholzer, 2003; BargasAvila et al., 2007; Jarrett and Gaffney, 2008).

Another recommendation is to maintain an intuitive question order, e.g. first ask for the name, then the address and at the end for the telephone number (Beaumont et al., 2002). The structure of a form might be organized analogous to a conversation: the content can be grouped in a logical way and might reflect users' understanding of the topic (Jarrett, 2000; Jarrett and Gaffney, 2008; Wroblewski, 2008). This can best be realized by mapping the virtual environment as closely as possible to the natural environment, one which is already familiar to users (Garrett, 2002). If they are familiar with a concept in real life, probably they will understand this concept if it is also applied to the online environment. In the case of web forms, this can for example be realized by providing labels and textboxes for answers in the same way as for paper forms. A study has shown, for example, that providing a dropdown menu for entering street type (e.g. road, street, avenue) caused people to turn back to the previous field where they had already entered it because they were accustomed to entering the street type with the street name (Nielsen, 2000).

Within online forms, labels have a direct influence on the form's usability (Beaumont et al., 2002). The position of labels relative to the input field was examined in eye-tracking studies (Penzo, 2006; Das et al., 2008). Penzo compared left-, right- and top-aligned labels and came to the conclusion that with left-aligned labels people needed nearly twice as long to complete the form as with right-aligned labels. Additionally, the number of fixations needed 
with right-aligned labels was halved compared to left-aligned labels. The fastest performance, however, was reached with topaligned labels, which required only one fixation to capture simultaneously both the label and the input field. As a result of this study, it is recommended to use left-aligned labels for unfamiliar data where one wants users to slow down and consider their answers. On the other hand, if the designer wants them to complete the form as quickly as possible, top-aligned labels are recommended (Wroblewski, 2008).

In an online form, only questions that really need to be answered should be asked (Jarrett and Gaffney, 2008). It is important to make clear in advance which fields are mandatory and which are optional (Linderman and Fried, 2004). Today, this is often realized through the use of an asterisk to indicate required fields. A study examined whether highlighting required fields by color coding leads to a faster completion time compared to the use of an asterisk next to required fields. It was discovered that people were faster at filling in the form, made fewer errors and were more satisfied when the required fields were highlighted in color (Pauwels et al., 2009). Another study found that people were fastest in filling in required fields when the required and optional fields were separated from each other (Tullis and Pons, 1997).

One of the key requirements to ensure high data quality is to provide the right input type, because these interface elements can strongly influence users' answers (Couper et al., 2004). Usual input types for filling out web forms are checkboxes, textboxes, radio buttons, and drop-down menus (Jarrett and Gaffney, 2008). Textboxes differ from the other response formats in their unlimited possibilities of answer options (free text entry). Users' preferred input types for providing answers online are textboxes (Beaumont et al., 2002). Open-ended questions have the advantage of discovering responses that individuals give spontaneously and avoiding the bias that may result from suggesting options to individuals, as may happen when using structured answer options. But in comparison to the latter, open-ended questions also have the disadvantage of extensive coding, larger item non-response, and more missing or inadequate data (Reja et al., 2003).

To reduce the negative impact of open-ended questions, website owners often impose specific format restrictions on users. They force for example the chosen password to have a minimum length or the provided date of birth to be in a specific format such as day-month-year. Little empirical research has been conducted to discover the most effective and understandable way of communicating these restrictions to users. Some authors simply suggest accepting entries in all common formats, because this saves users the trouble of trying to figure out which format is required (Linderman and Fried, 2004). If a defined format is required, sample entries can be provided to minimize confusion and support fast completion.

Users often take the size of a textbox to be an indication of the answer length that is expected from them (Beaumont et al., 2002; Wroblewski, 2008). Another study found that users gave more answers in the expected date format (two characters for the month and four for the year) if the field for the month was half the size as the one for the year rather than when both fields were the same size (Christian et al., 2007). People give more incorrect answers if the size of the input field does not fit the length of the expected input (Couper et al., 2001). Symbols (MM - YYYY vs. month - year) significantly increase the likelihood that respondents report their answer in the desired format. The symbols convey additional information to respondents (i.e., the number of digits expected) and communicate that information efficiently (Christian et al., 2007).

There are two important factors regarding the communication of format restrictions: timing and content.
(1) Form using no visual format restriction. Users are not informed in advance about the password policy (amazon.com).

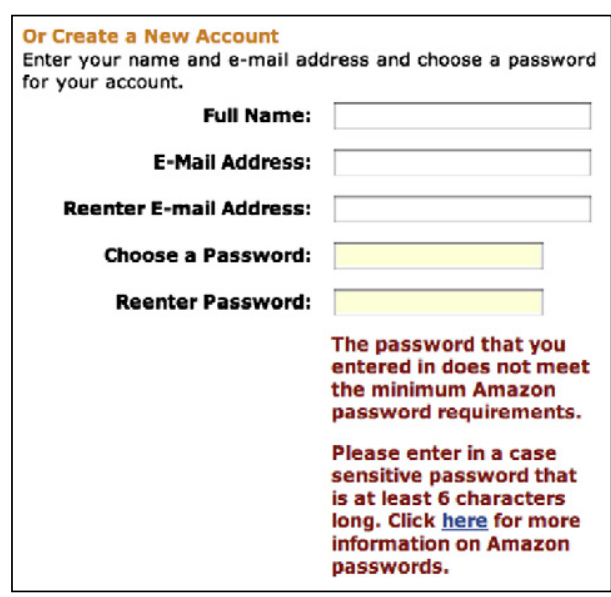

(3) Form using format specification. Users are told that the minimum length for the password is 8 characters (google.com).

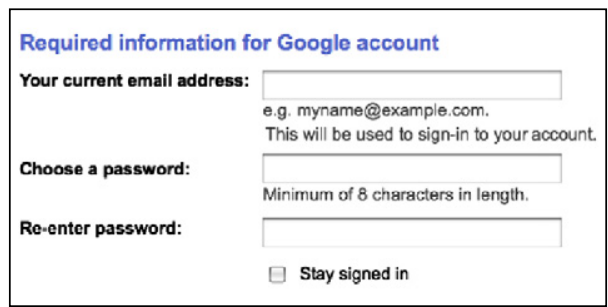

(2) Form using format example: Users are shown that the Yahoo ID equals the e-mail adress (yahoo.com)

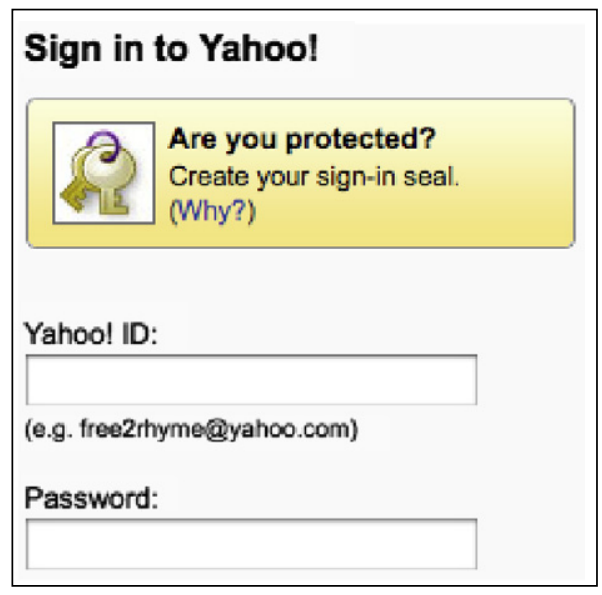

(4) Form using format example and specification. Users are informed about the password policy in detail (ebay.com).

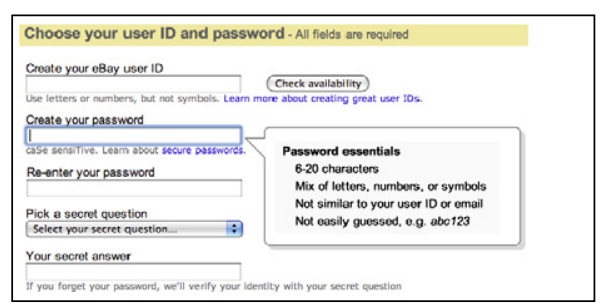

Fig. 1. Examples of various ways to communicate format restrictions to users from Bargas-Avila et al. (2010). 


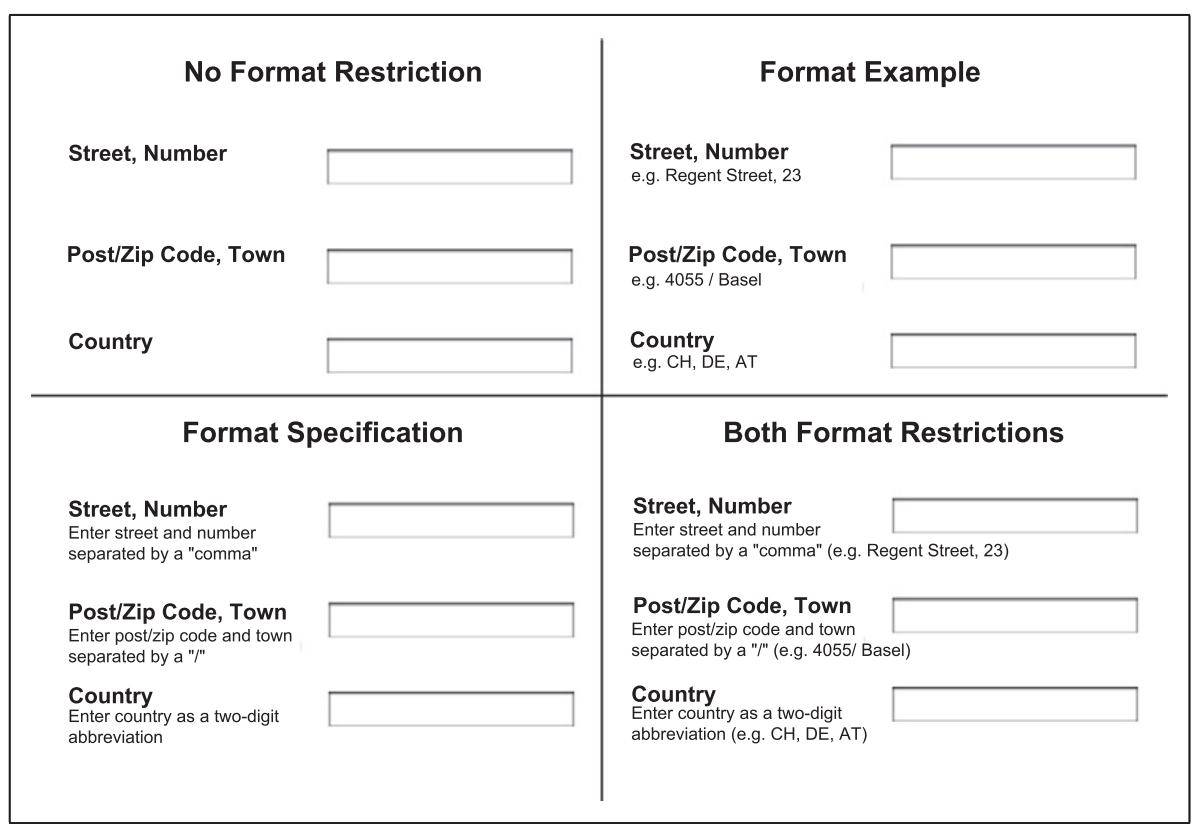

Fig. 2. Some questions used in this study (all four conditions; translated by the authors).

(1) Timing. Format restrictions can either be placed and communicated within the form in advance or appear as error messages after an erroneous input has been made. In most usability guidelines, error prevention is recommended as an important topic. If it is possible to guide users so that mistakes can be avoided, this should be done (see e.g. ISO-9241, 1996-2002; Stewart and Travis, 2003). Following this line of thought, it seems reasonable that format restrictions should be explicitly stated in advance to help instruct respondents to report their answers in the desired format. Helping respondents get it right the first time avoids error messages, reduces cognitive load (see e.g. Schmutz et al., 2009,2010) and frustration, and increases overall response efficiency.

(2) Content. Restrictions are usually communicated in two ways: the restriction can be stated as format specification (e.g. "Password must contain at least 8 characters and a number") or using a format example (e.g. "For example "passw0rd"."). Some designers prefer to combine format specifications and examples (here: "Password must contain at least 8 characters and a number, e.g. passw0rd").

Even though the ISO 9241-12 standard contains recommendations on how to present visual information on screens, so that users can easily perform perceptual tasks (ISO-9241, 1996-2002) such as in this case perceiving the entry restrictions of a specific field, currently there seems to be no consistent way of implementing timing and content of these format specifications in the World Wide Web. Fig. 1 shows that various examples can be found, even in major players like Amazon, eBay, Google or Yahoo. At the moment no work is known to the authors that empirically explores the optimal way of communicating input restrictions in online forms. The study presented here addresses this issue. Based on the assumption that format restrictions can be stated in advance (format restriction as part of the form/label) or after the error occurs (as an error message), and that they can be communicated using format specifications, format examples, or a combination of both, it is possible to study the following conditions (see Figs. 1 and 2):
(1) No visual format restriction: No information provided to users within the form - if the input is erroneous, users are informed with an error message that there are restrictions.

(2) Format example: Small amount of information provided to users via an example. The underlying rule is not explicitly stated, leaving room for interpretation and the necessity to deduce the rule.

(3) Format specification: More information provided to users via the explicit rule that must be fulfilled. No example is provided, leaving room for wrong applications of the rule.

(4) Format example and specification: Maximum amout of information provided to users. Explicit rule and an application of the rule in the form of an example helps users to understand and use the rule.

Following this line of thought, it can be hypothesized that format example and specification will perform best regarding number of erroneous entries and number of trials until the form is submitted successfully, because the maximum amount of information is provided to users. Format specification will outperform format example, because less interpretation and deduction is demanded from users, and no visual format restriction will perform worst, because no information at all is provided to users.

\section{Method}

To examine the usage of format restrictions in forms, an online experiment was conducted (German). A one-way between-subjects design was chosen in order to examine the four format restriction conditions: (1) no visual format restriction, (2) format example, (3) format specification, (4) format example and specification.

All participants were recruited via e-mail using the university recruitment database of people interested in taking part in studies. They were directly led to the online study by clicking on a link in the recruiting e-mail, where they had to complete an online form. A total of 169 subjects completed the experiment. Three participants were excluded because of invalid data (technical problems). 
Table 1

Format restrictions used for this experiment (translated by the authors).

\begin{tabular}{|c|c|c|}
\hline Field label & Format example & Format specification \\
\hline Street & e.g. Regent Street, 23 & $\begin{array}{l}\text { Enter street and number } \\
\text { separated by a "comma" }\end{array}$ \\
\hline Zip, Town & e.g. 8056/Zurich & $\begin{array}{l}\text { Enter post/zip code and town } \\
\text { separated by a "/" }\end{array}$ \\
\hline Country & e.g. $\mathrm{CH}, \mathrm{DE}, \mathrm{AT}$ & $\begin{array}{l}\text { Enter country as a two-digit } \\
\text { abbreviation }\end{array}$ \\
\hline Date of birth & e.g. $13-05-1966$ & $\begin{array}{l}\text { Date of birth in dd-mm-yyyy } \\
\text { format }\end{array}$ \\
\hline Phone & e.g. $061-9024568$ & $\begin{array}{l}\text { Enter area code and telephone } \\
\text { number separated by a "-" }\end{array}$ \\
\hline Username & e.g. user358 & $\begin{array}{l}\text { Username must be at least } 6 \\
\text { characters }\end{array}$ \\
\hline Password & e.g. ready 4 takeoff & $\begin{array}{l}\text { Password must contain at least } \\
\text { one number }\end{array}$ \\
\hline
\end{tabular}

The age of the remaining 166 participants ranged from 15 to 64 years with a mean of $27(S D=8.7)$; $59 \%$ were women. There were no significant differences regarding the distribution of age and sex between the experimental conditions. An MP3 player was raffled amongst all participants as a reward.

The core of the experiment was an online form presented on one page containing 15 fields that should be answered with personal data (e.g. name, address, hobbies, etc.). Seven fields were implemented with format restrictions and used for experimental manipulation. The conditions format example and format specification are listed in Table 1 . The condition format example and specification is a combination of both format restrictions and was shown in such a way that the format specification was followed by the format example in brackets (see Fig. 2).

On first sight of the online study, participants received a brief overview. They were informed that their personal data would be handled with discretion and that there was a possibility to take part in a raffle. Then they were randomly assigned to one of the four conditions and the main form was presented. After participants had filled in the fields and submitted the form for the first time, the seven fields were checked by the system and erroneous or incomplete fields were marked at once, displaying the format specification at the right side of each response field as an error message. This procedure was the same for all four conditions and was repeated until all questions were filled in correctly. As soon as the form was submitted successfully, the participants were thanked for their participation and could provide their e-mail ad- dress for the raffle. Some questions included in the online form can be seen in Fig. 2. Dependent variables were the number of errors until the online form was submitted the first time, the number of errors until the online form was submitted correctly and the number of trials until the online form was submitted correctly.

\section{Results}

The measured dependent variables of the four conditions are shown in Table 2. Where not stated otherwise, an $\alpha$ level of .05 was used for statistical tests.

In order to test whether the dependent variables differed between the format restrictions, a Kruskal-Wallis one-way ANOVA was conducted. The nonparametric test was chosen because none of the dependent variables were normally distributed. There was a significant main effect for all dependent variables. The number of errors up to the point that the online form was submitted the first time differed significantly $(H(3)=89.70, p<.001)$. The same was the case for the number of errors until the online form was submitted correctly $(H(3)=83.92, p<.001)$ and the number of trials until the online form was submitted correctly $(H(3)=29.73$, $p<.001$ ).

To further test the hypotheses, all conditions were compared. For this analysis, Mann-Whitney $U$ tests were used. Similar results could be observed for all dependent variables (see Table 3 ).

The results indicate that showing no visual format restrictions is less efficient than providing instructions. Comparing format examples with format specifications, the latter led to fewer errors and trials. Analogous results were found comparing format examples with the combination of both format restrictions. Analysis of the data also indicated that there is no significant difference between using format specifications and using both format restrictions.

For the upper overall analysis, the values for all input fields were pooled together. But the nature of the information requested for different input fields varied greatly. For example, asking for a username is a far more complex and challenging question than for example asking for the date of birth. When asked for date of birth, all users will have a convenient answer available in mind and will need only to consider whether they are willing to provide this information and the format in which it is required. By comparison, a username is far more demanding to users. They will have to think what username they will be able to recall easily, what usernames they use for other websites, what usernames might already be taken, whether the username will reveal their identity and

Table 2

Statistical parameters for different format restrictions.

\begin{tabular}{|c|c|c|c|c|}
\hline Measures & $\begin{array}{l}\text { No visual format restriction } \\
M(S D)\end{array}$ & $\begin{array}{l}\text { Format example } \\
M(S D)\end{array}$ & $\begin{array}{l}\text { Format specification } \\
M(S D)\end{array}$ & $\begin{array}{l}\text { Format example and specification } \\
M(S D)\end{array}$ \\
\hline First time errors & $5.5(.2)$ & $3.6(.3)$ & $1.3(.2)$ & $1.6(.2)$ \\
\hline Total no. of errors & $6.6(.3)$ & $4(.4)$ & $1.6(.3)$ & $1.8(.3)$ \\
\hline No. of trials & $2.3(.2)$ & $2.2(.1)$ & $1.9(.2)$ & $1.9(.1)$ \\
\hline
\end{tabular}

Table 3

Calculated contrasts between different format restrictions.

\begin{tabular}{|c|c|c|c|}
\hline Contrast & No. of errors until first submission & No. of errors until correct submission & No. of trials until correct submission \\
\hline No visual format restriction vs. Format example & $U(44,41)=340^{*}$ & $U(44,41)=335.5^{*}$ & $U(44,41)=631.5^{*}$ \\
\hline Format example vs. Format specification & $U(41,40)=302^{*}$ & $U(41,40)=338.5^{*}$ & $U(41,40)=580^{*}$ \\
\hline Format example vs. Both format restrictions & $U(41,41)=375.5^{*}$ & $U(41,41)=380.5^{*}$ & $U(41,41)=631.5^{* *}$ \\
\hline Format specification vs. Both format restrictions & $U(40,41)=691$ & $U(40,41)=715.5$ & $U(40,41)=762$ \\
\hline
\end{tabular}

${ }^{*} p<.01$, one-tailed test.

$p<.05$, one-tailed test. 


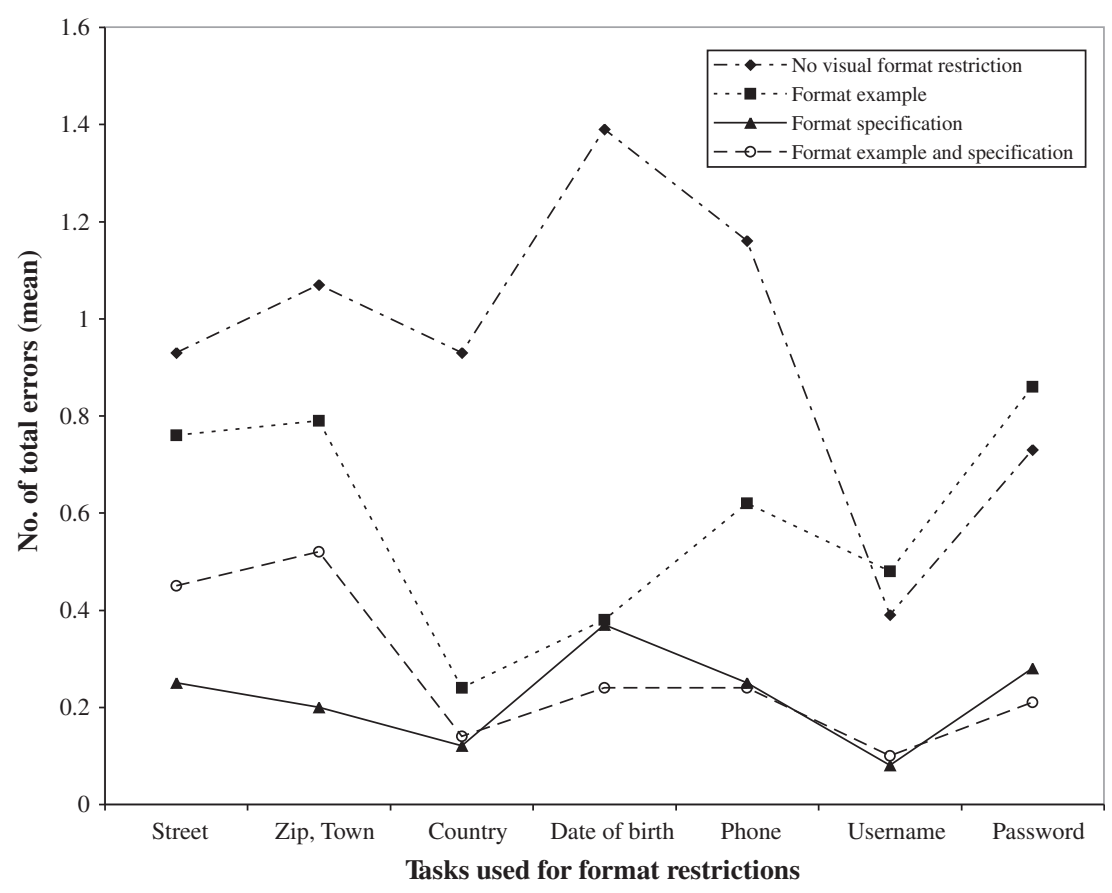

Fig. 3. Mean number of total errors for every task used.

Table 4

Statistical parameters for each task.

\begin{tabular}{|c|c|c|c|c|c|}
\hline Tasks & $\begin{array}{l}\text { No visual format restriction } \\
M(S D)\end{array}$ & $\begin{array}{l}\text { Format example } \\
M(S D)\end{array}$ & $\begin{array}{l}\text { Format specification } \\
M(S D)\end{array}$ & $\begin{array}{l}\text { Format example and specification } \\
M(S D)\end{array}$ & $H(3)$ \\
\hline Street & $.93(.26)$ & $.76(.48)$ & $.25(.44)$ & $.45(.50)$ & $47.24^{*}$ \\
\hline Zip, Town & $1.07(.33)$ & $.79(.42)$ & $.20(.46)$ & $.52(.63)$ & $59.04^{*}$ \\
\hline Country & $.93(.55)$ & $.24(.43)$ & $.12(.34)$ & $.14(.42)$ & $65.35^{*}$ \\
\hline Date of birth & $1.39(1.15)$ & $.38(.58)$ & $.37(.59)$ & $.24(.48)$ & $64.64^{*}$ \\
\hline Phone & $1.16(1.10)$ & $.62(.62)$ & $.25(.71)$ & $.24(.48)$ & $51.20^{*}$ \\
\hline Username & $.39(.54)$ & $.48(.74)$ & $.08(.27)$ & $.10(.30)$ & $19.66^{*}$ \\
\hline Password & $.73(.66)$ & $.86(.84)$ & $.28(.78)$ & $.21(.42)$ & $32.99^{*}$ \\
\hline
\end{tabular}

* $p<.01$.

whether they are willing to reveal it. A further aspect is the fact that the restrictions that are demanded can vary greatly. So, for example, the rule that users have to apply to format a date correctly (e.g. dd-mm-yyyy) is very different when compared to using a two-digit abbreviation for a country. All these differences may interfere with the perception of format restrictions and may even have an influence on error and trial rates. Therefore an analysis on task level was performed to explore whether there are differences between the chosen tasks.

To test whether the tasks differ regarding total number of errors, a Friedman ANOVA was performed. The nonparametric test was chosen because some of the dependent variables were not normally distributed. Results indicate that there are significant differences for the format restrictions 'No visual format restriction' $\left(\chi_{r}^{2}(6)=69.198, p<.001, N=44\right)$, 'Format example' $\left(\chi_{r}^{2}(6)=\right.$ $52.920, p<.001, N=41)$, and 'Format example \& specification' $\left(\chi_{r}^{2}(6)=33.572, p<.001, N=40\right)$, whereas no difference can be stated for 'Format specification' $\left(\chi_{r}^{2}(6)=11.952, p<.063, N=41\right)$. Fig. 3 shows the mean number of total errors for every task. There are not only differences between the format restrictions used, but also between the different tasks. Kruskal-Wallis one-way ANOVA indicates that the factor task shows significant differences for all tasks (see Table 4).

\section{Discussion}

This study sheds light on the question of whether format restrictions should be communicated to Internet users and shows how these restrictions can be formulated.

The results clearly show that it is crucial to inform users in advance that format restrictions will be imposed. Using visual format restrictions led to significantly fewer errors and trials, thus enhancing and supporting fluent form interaction. In view of these results, it is interesting that so many forms are still implemented without a clear communication of format restrictions, leading to delayed or maybe even aborted interactions.

With regard to the formulation of these format restrictions (content), it was shown that it is better to state a clear and simple rule (format specification) that helps users to understand how they are meant to format their response. Users seem to be able to apply these rules to their own situation and provide the correct answer. Using format examples alone, on the other hand, seems to leave too much room for interpretation, leading to more errors and trials. At this point, all hypotheses could be confirmed: Using no visual format restrictions performed worst, using format examples led to fewer errors, and using format specifications led to an even better performance. 
Concerning the combination of format examples and specifications, it was hypothesized that this condition would lead to even fewer errors than using only format specifications. The example would lead users to understand the rule better and enhance their interpretation and interaction. This hypothesis was not confirmed: Using examples did not further support users in avoiding mistakes. Providing an additional example neither helps nor constrains users, but discarding examples may provide website developers with more room for clear and simple screen designs. In addition to this advantage, the detailed data analysis on task level showed that only the condition using format restrictions without examples revealed no task-specific differences. It seems that the use of format specifications without an additional example helps to level out task differences a little better when compared to the other format restriction methods.

Fig. 3 shows that there are also task specific differences. On the one hand users show for example major problems in formatting the password correctly, even when format restrictions are used. On the other hand, it was quite easy to enter the country in a two-digit abbreviation; users had few problems in understanding the underlying rule. It is quite common to use these abbreviations in Europe, a fact that probably helped users to understand the rule and apply the correct formatting. Indeed works show the importance of considering cultural factors when it comes to web form design (Recabarren et al., 2008; Recabarren and Nussbaum, 2010). The analysis on task level shows very clearly, that the difficulty of entering correct answers does not only depend on the information provided via format restriction labels, but also on the implied restriction on the data. Not all format restrictions used nowadays are made to control that the entered data is correct (e.g. e-mail address must use an @, zip code must contain 5 characters for the USA, date must be formatted in a certain way to avoid datemonth confusion). Sometimes restrictions are used solely to ensure consistent formatting in the companies' databases (e.g. formatting of the phone number or street name and number). The differences on task level indicate, that website developers should consider very carefully if a restriction is really necessary. Even with the best methods to communicate format restrictions users made mistakes - errors that slow users down and may even lead to dropouts. The imposed rules of this study were somewhat artificial and not conceived to analyze what kind of rules are easy or difficult to understand. Future studies may focus on restriction types and formulations, leading to precise recommendations for website developers.

Summing up, this study shows that the modification of a small text interface element has a measurable influence on error rate in online forms. Similar findings for text elements within websites were reported in the past (e.g. Chadwick-Dias et al., 2002). This knowledge may help website developers in supporting their users with little effort, because the clear and simple communication of format restrictions normally does not lead to a major design effort.

This study helps to clear up an important point regarding the design of usable forms: How should format restrictions be presented to users to reduce errors? At the same time it must be stressed, that the presented findings should be regarded as a first step. The study was conducted in a rigorous experimental setting. The chosen tasks were adapted to these conditions and not embedded in a real setting, such as a shopping or registration process. The ecological validity of the presented findings is therefore low. To overcome this limitation, future studies may vary interface elements within a real setting using real tasks. It remains to be seen if such an ecologically more valid setting reveals conflicting factors like e.g. screen real estate that interfere with the given recommendations.

Regarding the future outlook concerning usable forms, there are many open questions that must be answered. In recent years, new developments, like for example Web 2.0, have led to new ways of implementing interactions on the Internet. Nowadays, for example, users get more and more accustomed to receiving immediate feedback in webforms through the use of Ajax technology. While these interactional aspects were not covered in this publication, further studies are needed to understand how these new interaction styles can be implemented best, if we want to continuously enhance online forms in the future.

There is a growing body of empirical research and best practice recommendations by usability experts to achieve usable forms on the World Wide Web. Most studies - like the one presented here choose to explore one specific aspect or interface element to find the optimal solution. Usually this is done in a laboratory situation, using abstract forms with artificial tasks. In the near future, this knowledge needs to be consolidated in practical guidelines like e.g. the 20 Guidelines for Usable Web Form Design (Bargas-Avila et al., 2010). These guidelines must be empirically tested using real forms in realistic usage situations, to see whether they really fit users' mental models Roth et al., 2010 of a usable form and lead to better usability, manifested in faster form-completion time, fewer errors, higher user satisfaction and reduced dropout rate.

\section{Acknowledgments}

The authors would like to thank Peter Schmutz and Stefan Pauwels for the programming of this online experiment and all participants for their valuable contribution to this study.

\section{References}

Bargas-Avila, J., Oberholzer, G., 2003. Online form validation: don't show errors right away. In: Rauterberg, M., Menozzi, M., Wesson, J. (Eds.), HumanComputer Interaction INTERACT '03. IOS Press, Amsterdam, pp. 848-851.

Bargas-Avila, J., Oberholzer, G., Schmutz, P., de Vito, M., Opwis, K., 2007. Usable error message presentation in the world wide web: do not show errors right away. Interacting with Computers 19 (3), 330-341.

Bargas-Avila, J., Brenzikofer, O., Roth, S., Tuch, A., Orsini, S., Opwis, K., 2010. Simple but crucial user interfaces in the world wide web: introducing 20 guidelines for usable web form design. In: Matrai, R. (Ed.), User Interfaces. INTECH, pp. 1-10.

Beaumont, A., James, J., Stephens, J., Ullman, C., 2002. Usable Forms for the Web. Glasshaus, Birmingham.

Camenisch, J., Shelat, A., Sommer, D., Zimmermann, R., 2006. Securing user inputs for the web. In: Proceedings of the Second ACM Workshop on Digital Identity Management. ACM, pp. 33-44.

Chadwick-Dias, A., McNulty, M., Tullis, T., 2002. Web usability and age: how design changes can improve performance. ACM SIGCAPH Computers and the Physically Handicapped, 30-37.

Christian, L., Dillman, D., Smyth, J., 2007. Helping respondents get it right the first time: the influence of words, symbols, and graphics in web surveys. Public Opinion Quarterly 71 (1), 113-125.

Couper, M., Traugott, M., Lamias, M., 2001. Web survey design and administration. Public Opinion Quarterly 65 (2), 230-253.

Couper, M., Tourangeau, R., Conrad, F., Crawford, S., 2004. What they see is what we get: response options for web surveys. Social Science Computer Review 22 (1) 111-127.

Das, S., McEwan, T., Douglas, D., 2008. Using eye-tracking to evaluate label alignment in online forms. In: Proceedings of the 5th Nordic Conference on Human-Computer Interaction: Building Bridges. ACM, New York, NY, USA, pp. 451-454.

Garrett, J., 2002. The Elements of User Experience. New Riders Indianapolis, NY, USA.

ISO-9241, 1996-2002. Ergonomic requirements for office work with visual display terminals (parts $1-17$ ).

Jarrett, C., 2000. Designing usable forms: the three-layer model of the form. Tech. rep., Effortmark.

Jarrett, C., Gaffney, G., 2008. Forms that Work: Designing Web Forms for Usability Morgan Kaufmann, MA, USA.

Linderman, M., Fried, J., 2004. Defensive Design for the Web: How to Improve Error Messages, Help, Forms, and Other Crisis Points. New Riders Publishing, Thousand Oaks, CA, USA.

Nielsen, J., 2000. Drop-down menus: Use sparingly. <http://www.useit.com/ alertbox/20001112.html>.

Nielsen, J., 2005. Forms vs. applications. <http://www.useit.com/alertbox/ forms.html>.

Pauwels, S., Hübscher, C., Leuthold, S., Bargas-Avila, J., Opwis, K., 2009. Error prevention in online forms: use color instead of asterisks to mark required fields. Interacting with Computers 21 (4), 257-262. 
Penzo, M., 2006. Label placement in forms. <http://www.uxmatters.com/MT/ archives/000107.php>.

Recabarren, M., Nussbaum, M., 2010. Exploring the feasibility of web form adaptation to users' cultural dimension scores. User Modeling and UserAdapted Interaction 20 (1), 87-108.

Recabarren, M., Nussbaum, M., Leiva, C., 2008. Cultural divide and the internet. Computers in Human Behavior 24 (6), 2917-2926.

Reja, U., Manfreda, K., Hlebec, V., Vehovar, V., 2003. Open-ended vs. close-ended questions in web questionnaires. Advances in Methodology and Statistics 19 159-177.

Roth, S., Schmutz, P., Pauwels, S., Bargas-Avila, J., Opwis, K., 2010. Mental models for web objects: where do users expect to find the most frequent objects in online shops, news portals, and company web pages? Interacting with Computers 22 (2), 140-152. <http://dx.doi.org/10.1016/j.intcom.2009.10.004>.
Schmutz, P., Heinz, S., Métrailler, Y., Opwis, K., 2009. Cognitive load in ecommerce applications - measurement and effects on user satisfaction. Advances in Human-Computer Interaction Volume 2009, 9 (article ID 121494).

Schmutz, P., Roth, S., Seckler, M., Opwis, K., 2010. Designing product listing pages effects on sales and users' cognitive workload. International Journal of HumanComputer Studies 68 (7), 423-431.

Stewart, T., Travis, D., 2003. Guidelines, standards, and style guides. In: Jacko, J.A., Sears, A. (Eds.), The Human-Computer Interaction Handbook. Lawrence Erlbaum Associates, London, pp. 991-1005.

Tullis, T., Pons, A., 1997. Designating required vs. optional input fields. In: Conference on Human Factors in Computing Systems. ACM, New York, NY, USA, pp. 259-260.

Wroblewski, L., 2008. Web Form Design: filling in the blanks. Rosenfeld Media, Brooklyn, New York 